\title{
GRADATION, COLOUR RANGE AND COLORIMETRIC ACCURACY OF DIGITAL PROJECTOR JVC DLA-RS 15
}

\author{
Iva Molek ${ }^{1}$ (D), Dejana Javoršek ${ }^{2}$ (iD \\ ${ }^{1}$ Multimedia and Graphic Technology Secondary School, Ljubljana, Slovenia \\ ${ }^{2}$ University of Ljubljana, Faculty of Natural Sciences and Engineering, Ljubljana, Slovenia
}

\begin{abstract}
Nowadays, digital projectors are used for displaying static and dynamic content, while operating with a wide range of different projection technologies. Presentation methods are also used to present important colour reproductions where the colours should be displayed accurately. The purpose of our research was to improve the colour quality of presentation and to define display of colour in digital projection. For that purpose high quality digital projector JVC DLA-RS15 was used. According to standard ISO 12646: 2010, was researched the display of colours in digital projection. For the colorimetric analysis of the displayed colours, the spectrophotometer i1Pro and the spectroradiometer PR-650 were used. Since the vast majority of digital photographs is captured and displayed in the standardized $s R G B$ colour space, we investigated how well the SRGB colour space is displayed using JVC DLA-RS15 digital projector and whether the standard could be achieved by profiling its modules. Based on the results of our research, it was found out that spectroradiometer PR-650 always shows the same results regardless of the measuring distance. However, in case of the spectrophotometer i1Pro, it proved best to perform measurements and profiling so that the measuring distance equals to the projection distance. The monitoring and the evaluation of digital projections based on chromaticity, graduation, channel and grey balance and colour range according to the standard ISO 12646:2010 is quite demanding and timeconsuming. It has also been found that a large colour gamut of saturated primary colours at digital projector does not guarantee the quality of the displayed colours in the standard SRGB colour space.
\end{abstract}

Key words: digital projector, colorimetric analysis, profiling, colours

\section{INTRODUCTION}

Projectors have become an important part of every serious presentation. Nowadays digital projectors are used to project static and dynamic contents, and function with the help of a wide array of various projection technologies and modules respectively. With the aid of the digital projection modules we can choose from different pre-set options; some projectors can be geometrically calibrated (manual setting of contrast, brightness, correlated colour temperature) and characterized (Ashdown et al, 2018; Cooper, 2016; Matt, 2003; Wikipedia, 2018). In reference to the testing of digital projectors, only the source (Park \& Park, 2010) is summarized. According to the ISF (Turk, 2018) specification for certification, the most precise rendering of six colours has been achieved so far with a colour difference of 1-3 with the default settings and a colour difference of 0.5 with the created profile (for all six re-produced colours) with the JVC DLA-RS25 digital projector. The source does not specify the details of the test methods, the method of measurement, or the calculation of the colour difference - the only information that is given is that the ISF calibrator is certified (Turk, 2018).

Experience and some sources (Matt, 2003) suggest that the calibration and profiling of a digital projector with visual evaluation can improve the display of colours. Other sources (Turk, 2018) indicate excellent results, but they probably only apply to the six basic colours, whereas there is no source that would deal with objective colorimetric evaluation of a digital projection based on the representation of real colours in different observation conditions.

The purpose of our research was to improve the colour quality of presentation and to define display of colour in digital projection. For that purpose high quality digital projector JVC DLA-RS15 was used.

The JVC DLA-RS15 belongs to the category of home theatre projectors (Turk, 2018; Feierman, 2018). It is based on liquid crystal technology on LCoS silicon. The rendering resolution of the projector is very high, up to $1920 \times 1200$ pixels, if such resolution is also supported by the computer. The projector has six modules for watching movies, i.e. Cinema 1, Cinema 2, Cinema 3, Natural, Stage and Dynamic. In the projector menu, a colour temperature of 5800 K, 6500 K, 7500 K, 9300 K, High Bright, Custom 1, 2, 3 (custom settings) can also be determined within the individual modules. The projector menu also includes gamma and grey (channel) balance settings. The JVC DLA-RS15 does not have full support for colour management (CMS); it only allows calibration of the channel (grey) level. 


\section{EXPERIMENTAL PART}

For measurements of projected colours according to standard SIST ISO 12646:2010 (Feierman, 2018), was used: following equipment: digital projector JVC DLA-RS15, personal computer with Power Point, Reflecta projection, spectrophotometer i1Pro placed on a photographic tripod with applications i1Share and KeyWizard, spectroradiometer PR-650 placed on a photographic tripod and measurement charts according to standard. Standard ISO 12646:2010 (Graphic technology - Displays for colour proofing Characteristics and viewing conditions) (Cooper, 2016) includes provisions for observing conditions in screen preview of printed material and soft proofing respectively. Because of the extensiveness of the standard, merely the measurements of gradation, colour range and colorimetric accuracy are focused on and performed a test of light conditions prior to measurements and profiling. Tests were performed in studio of Multimedia and Graphic Technology Secondary School.

First, the light conditions were tested. The white screen DPT-W315 was projected onto the projection screen. The brightness was measured in the middle of the projection, and on the left and the right for half the width of the projection outside the screen. The condition of the brightness (LV) of the external field surrounding the screen was always fulfilled irrespective of the instrument, the measurement distance and the spatial conditions. With the already projected measuring plate (DPT-W315), we also measured the brightness (LV) of the inner white field, i.e., the projection screen without projection. This condition was always fulfilled. Then the colour plate DPT-K315 was projected. The brightness was measured in the middle of the projection, and then the digital projector was turned off and measured the brightness again. With the JVC DLA-RS15, the standard was fully complied with.

After 45 minutes of heating the projector measurements of the unprofiled projection were performed. Calibration and profiling of the digital projector were performed using well-established applications i1Match 3.6 and basICColor display 4.1.22 in combination with spectrophotometer EyeOne Pro (i1Pro). In the measurements, we focused on the default and reset projection modules of the selected projector:

- $\quad$ Natural Module: CCT: 6500 K, Gamma 1.0, Diaphragm 3

- Natural HB: Contrast: default, CCT: High Bright, Flash: 3, Bulb: High (200 W), Gamma: normal

- Natural 2.2: Contrast is default, CCT: 6500 K, Flash: 3, Bulb: High (200 W), Gamma: 2.2

- User1: Contrast: +50, CCT: High Bright, Flash: 3, Gamma: normal (Park et al, 2010)

- User-2: Contrast: -1 Brightness: 1 Saturation: -12 Hue: 0, CCT (gain R=9, G = -7, B = -21; -1), Gamma: normal, Flash: 3, Bulb: High (200 W) (Park et al, 2010)

Prior to each measurement, the DPT-W315-T colour plate was projected on the projection screen, and instantly measured using the instrument with the target in the middle of the central field 5. We started with gradation measurements by projecting the colour plates (Figures $1 \mathrm{a}$ and $1 \mathrm{~b}$ ) of the centred grey fields (DPT-gamaL (100-0) has 21 centred grey fields in the range $R=G=B$ with values from 255 to 0 ) one by one to the projection screen. We measured the standardized colour values $X, Y, Z$, and calculated the gamma, which should be in the range of 1.8-2.4.

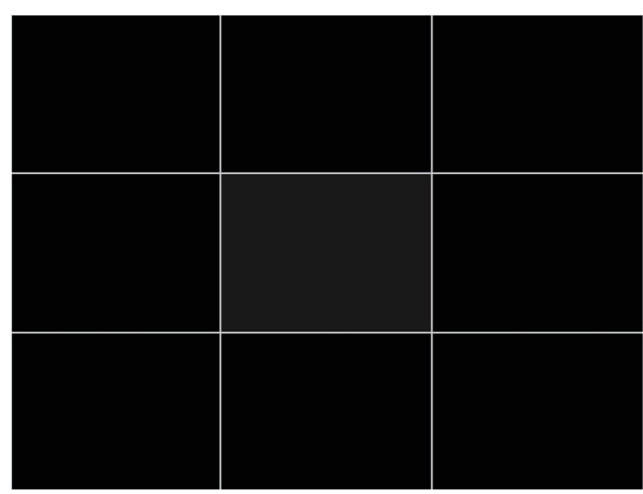

a)

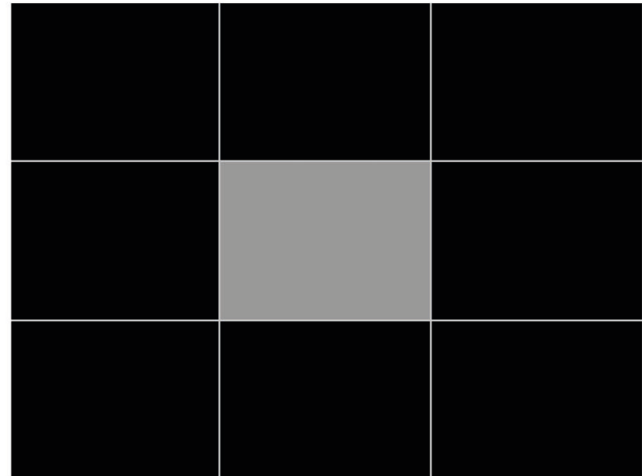

b)

Figure 1: Colour chart for measuring gradations: a) DPT-gammaL (25) and b) DPT-gammaL (135)

For defining the colour range of the digital projector, the DPT-SPC colour charts (Fig. 2a and $2 \mathrm{~b}$ ) were projected one after the other (DPT-SPC colour plates have eight centred fields with primary R, G, B and secondary C, M, Y colours, including white colour W and black colour K. On each one, the standardized 
colour values of $X, Y, Z$ of the central field were measured. The chromatic coordinates $x, y$, and $u^{\prime}, v^{\prime}$ were calculated, so that the brightness of white colour is defined with $L^{*}=100$.

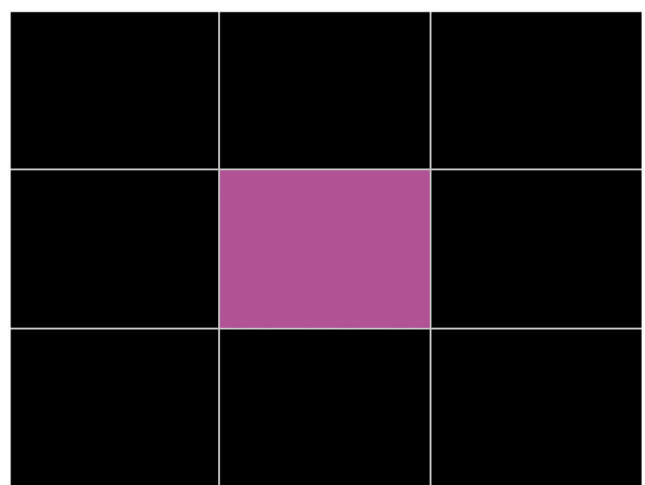

a)

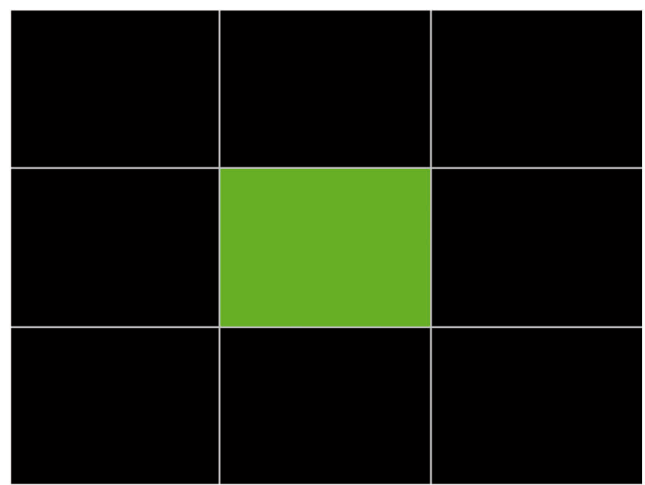

b)

Figure 2: The colour charts for measuring the gamut: a) magenta and b) green.

The colorimetric accuracy of the digital projection was evaluated by using the 24 fields of the Colour Checker Classic colour chart. One after another, colour charts were projected to the screen (Figures $3 a$ and $3 \mathrm{~b}$ ) and standardized colour values $X, Y, Z$ of the central field were measured on each of them. Colour differences $\mathrm{dE}^{*} \mathrm{ab}$ between the displayed and reference values were calculated.

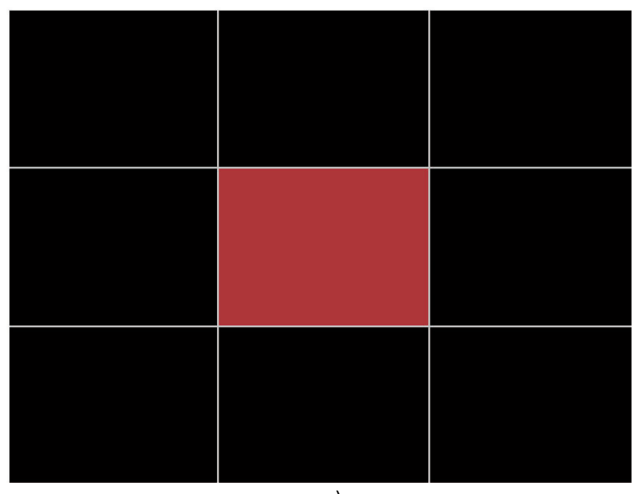

а)

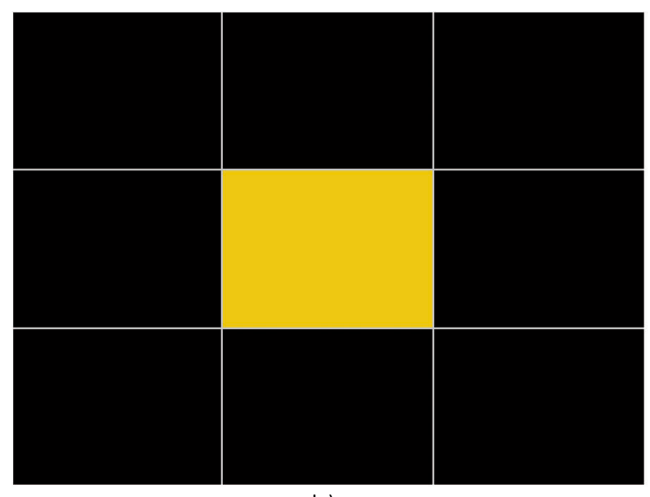

b)

Figure 3: Colour Checker Classic colour charts a) Patch 15 Red and b) Patch 18 yellow

\section{RESULTS AND DISCUSSION}

\section{1 Gradation}

In all projection modules, except in User-1, gamma is within the desired range, as can be seen in Figure 4. Since the projector settings also allow the gamma to be adjusted, an additional test with reset gamma 2.2 was performed, the Natural 2.2 module. It turned out that this setting had no effect neither on the value of the gamma nor on the display of tones, i.e., gradation. An essential change is noted in gradation in the User-1 module with the High Bright white tile reciprocal temperature and the increased contrast +50 , which was set to ensure the highest possible brightness (LV) of the white tile and thus approximate the requirements of the SRGB standard, however, such settings lead us to unwanted display of tones. 


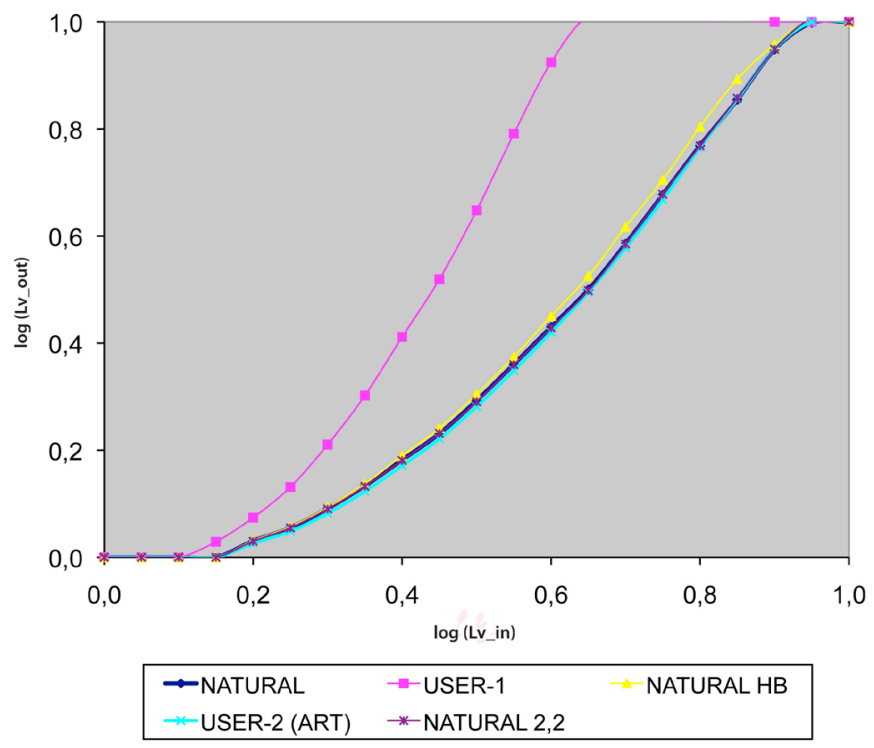

Figure 4: Gradation of the digital projector JVC DLA-RS15.

\subsection{Colour gamut}

It is clear from Figure 5 that all JVC DLA-RS15 digital projector modules feature exceptionally large colour scale, substantially larger than the standardized SRGB colour scale (sRGB: IEC mark). In Figure 6 in the diagram $L^{*}\left(C^{*} \mathrm{uv}\right)$ in yellow colour, we see that the colour is very saturated and almost as bright as white. All these are the consequences of the noticeably low brightness (LV) of the JVC DLA-RS15 digital projector.

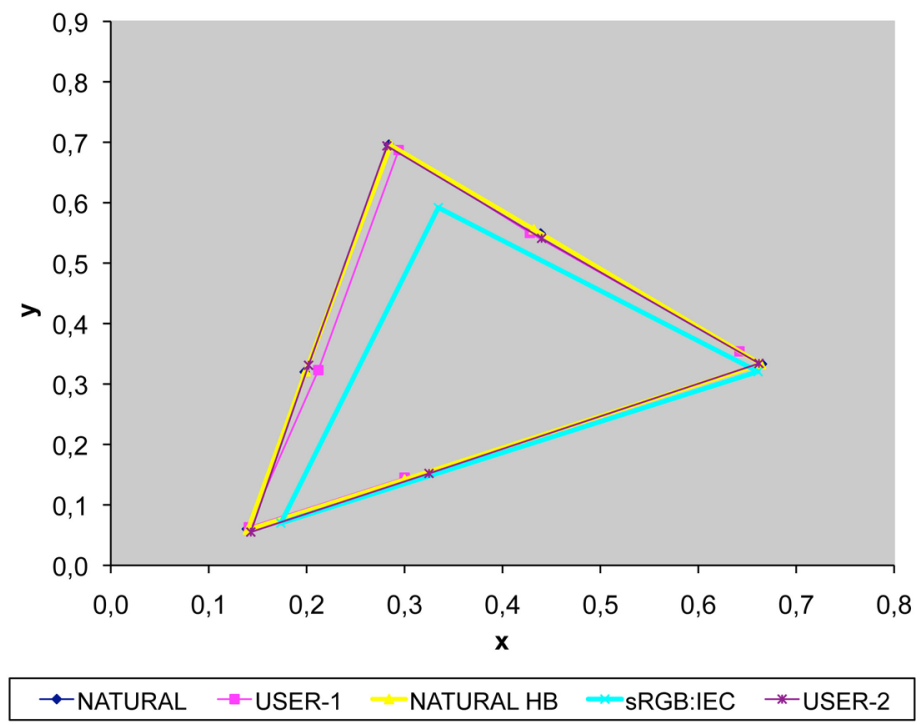

Figure 5: Colour gamut of the digital projector JVC DLA-RS15 presented CIE 1931 x,y chromatic diagram. 


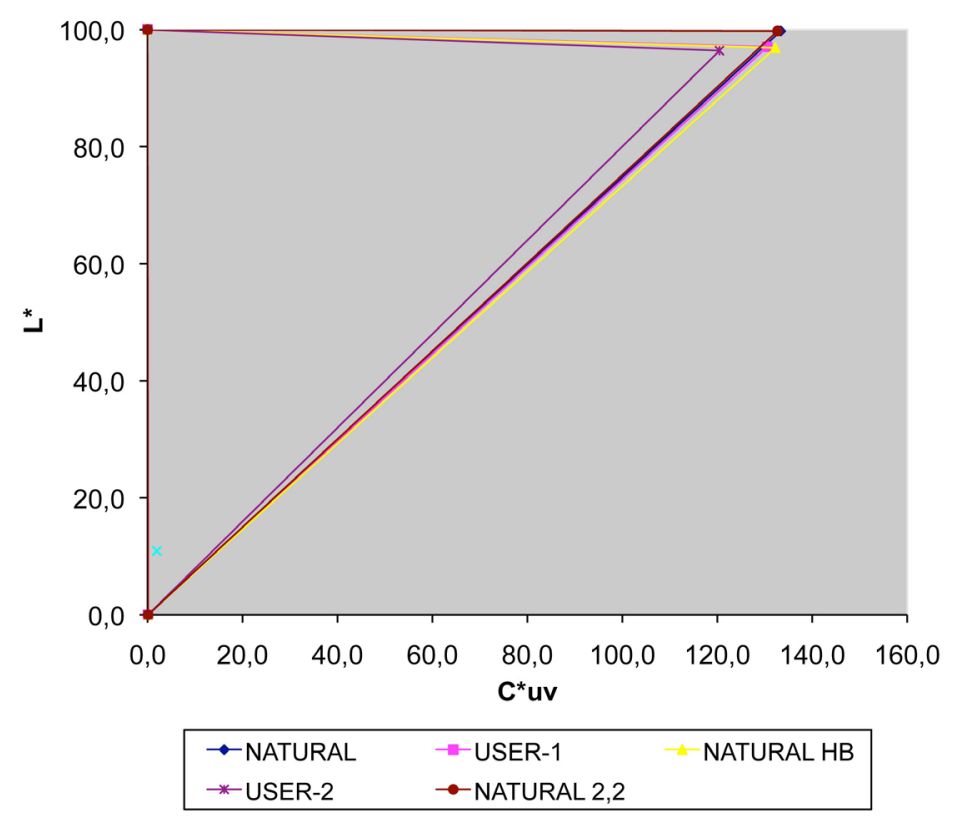

Figure 6: Brightness in relation to chrome for yellow of the digital projector JVC DLA-RS15.

\section{3 Colorimetric accuracy according to the ISO 12646:2010 Standard}

Table 1 shows the results of the measurements using the spectroradiometer and the spectrophotometer. In measurements with the spectroradiometer, it was found that the colours were displayed best in the Natural module with an average $\mathrm{dE}^{*} \mathrm{ab} 11.83$ and a maximum $\mathrm{dE}^{*} \mathrm{ab}$ 30.04. As expected, the colours were displayed worst in the User-1 module with an average $\mathrm{dE}^{*} \mathrm{ab} 23.0$ and the maximum dE*ab 58.0. In the spectrophotometer measurements we found that Colour Checker Classic colours are best shown in User-2 module with an average $\mathrm{dE}^{*} \mathrm{ab} 11.72$ and a low maximum dE*ab 17.58. They are followed by the default Natural module with an average dE*ab 13.05 and the maximum dE*ab 35.22, and the Natural HB module with an average $\mathrm{dE}^{*} \mathrm{ab} 14.54$ and the maximum $\mathrm{dE}^{*} \mathrm{ab} 40.32$.

This was followed by profiling the Natural HB module with different combinations of software and measurement technology. The Natural HB module was chosen to continue the tests, because it displays the tones somehow brighter than other modules. Measurements were performed with a spectroradiometer and spectrophotometer.

It is apparent from Table 2 that no combination of an application or a programme and a profiling instrument substantially improves the average colour deviation that, when measured with a spectroradiometer in the Natural HB module, comes to an average of $\mathrm{dE}^{*} \mathrm{ab} 12.7$ and a maximum of $\mathrm{dE}^{*} \mathrm{ab}$ 41.09. It is slightly exceeded by the colour profile of basICC display 4.1.22 + i1Pro with an average $\mathrm{dE}^{*} \mathrm{ab} 12.0$ and a maximum $\mathrm{dE}^{*} \mathrm{ab}$ 24.7, which is better compared to the maximum $\mathrm{dE}^{*} \mathrm{ab} 41.1$ without profile (Natural HB without profile). In both cases, the yellow colour in the field 16 with an average dE*ab 41.09 without the profile and with an average $\mathrm{dE}^{*} \mathrm{ab} 24.67$ with the basICC display $4.1 .22+\mathrm{i} 1$ Pro profile deviates the most. The situation remains the same when measuring the displayed colours with the spectrophotometer. The results are shown in Table 3, except that the average $\mathrm{dE}^{*}$ ab values of the achieved colour differences are slightly higher: the colour profile basICC display 4.1.22 + i1Pro again performs best with an average $\mathrm{dE}^{*} \mathrm{ab} 15.73$ and a maximum $\mathrm{dE}^{*} \mathrm{ab}$ 26.37. 
Table 1: The colorimetric accuracy of digital projector JVC DLA-RS15 module, evaluated by Color Checker Classic chart, measured by PR-650 and i1Pro; yellow colour marks the $d E^{*}$ ab values, lower than 5

\begin{tabular}{|c|c|c|c|c|c|c|c|c|}
\hline \multirow{2}{*}{ Color Checker Classic } & \multicolumn{4}{|c|}{$\Delta E^{*} a b ; P R-650$} & \multicolumn{4}{|c|}{$\Delta E^{*} a b ;$ i1Pro } \\
\hline & Natural & User-1 & Natural HB & User-2 & User-1 & Natural & User-2 & Natural HB \\
\hline Polje 1 ( Dark skin) & 7.47 & 12.39 & 4.03 & 7.42 & 8.89 & 8.44 & 7.38 & 11.36 \\
\hline Polje 2 ( Light skin) & 9.66 & 25.01 & 5.23 & 10.02 & 23.61 & 10.94 & 10.31 & 14.49 \\
\hline Polje 3 ( Blue Sky) & 8.48 & 21.48 & 9.90 & 9.03 & 18.69 & 9.54 & 10.93 & 10.11 \\
\hline Polje 4 ( Olive Green) & 9.07 & 22.96 & 9.44 & 8.71 & 19.19 & 10.32 & 9.83 & 11.69 \\
\hline Polje 5 ( Violet) & 8.19 & 19.95 & 8.99 & 9.51 & 18.34 & 9.30 & 11.91 & 10.43 \\
\hline Polje 6 ( Bluish Green) & 12.95 & 17.01 & 10.94 & 11.80 & 12.85 & 11.88 & 13.17 & 10.53 \\
\hline Polje 7 ( Orange) & 18.85 & 42.23 & 20.19 & 13.62 & 39.69 & 18.65 & 11.17 & 19.60 \\
\hline Polje 8 ( Purplish Blue) & 7.55 & 19.07 & 10.25 & 9.83 & 17.90 & 9.27 & 12.98 & 9.84 \\
\hline Polje 9 ( Moderate Red) & 6.53 & 18.29 & 7.51 & 9.80 & 18.21 & 8.79 & 9.11 & 13.40 \\
\hline Polje 10 ( Purple) & 9.29 & 25.07 & 13.38 & 7.68 & 20.41 & 11.20 & 8.86 & 13.86 \\
\hline Polje 11 ( Yelow Green) & 16.00 & 22.08 & 18.52 & 11.41 & 20.72 & 16.88 & 16.47 & 17.10 \\
\hline Polje 12 ( Orange Yellow) & 25.61 & 57.99 & 31.80 & 16.10 & 56.03 & 24.99 & 14.03 & 30.12 \\
\hline Polje 13 (Blue) & 8.77 & 29.39 & 14.76 & 8.46 & 23.38 & 10.42 & 13.45 & 11.35 \\
\hline Polje 14 ( Green) & 11.83 & 32.48 & 13.97 & 10.59 & 27.19 & 11.93 & 13.43 & 12.30 \\
\hline Polje 15 ( Red) & 11.70 & 13.22 & 6.97 & 9.03 & 8.62 & 10.88 & 8.41 & 9.03 \\
\hline Polje 16 ( Yelow) & 30.04 & 40.76 & 41.09 & 38.09 & 39.99 & 35.22 & 17.58 & 40.23 \\
\hline Polje 17 ( Magenta) & 7.66 & 24.48 & 12.15 & 9.46 & 23.04 & 10.34 & 13.27 & 15.33 \\
\hline Polje 18 ( Cyan) & 5.52 & 24.30 & 6.64 & 8.74 & 20.63 & 5.30 & 11.46 & 13.56 \\
\hline Polje 19 ( White) & 20.97 & 15.16 & 15.86 & 18.67 & 18.45 & 20.12 & 16.10 & 20.10 \\
\hline Polje 20 ( Neutral 8) & 13.93 & 8.02 & 10.98 & 11.84 & 9.21 & 13.51 & 13.41 & 13.86 \\
\hline Polje 21 ( Neutral 6,5) & 10.55 & 18.70 & 10.22 & 9.37 & 16.51 & 10.60 & 11.37 & 12.07 \\
\hline Polje 22 ( Neutral 5) & 8.16 & 19.71 & 8.88 & 7.93 & 15.83 & 9.12 & 9.39 & 10.74 \\
\hline Polje 23 ( Neutral 3,5) & 6.83 & 15.25 & 6.79 & 7.94 & 12.60 & 8.97 & 8.08 & 11.07 \\
\hline Polje 24 ( Black) & 8.39 & 6.23 & 6.21 & 9.68 & 9.79 & 16.67 & 9.23 & 16.76 \\
\hline \multicolumn{9}{|c|}{ All colours } \\
\hline$M x^{1} d^{*} a b$ & 11.83 & 22.99 & 12.69 & 11.45 & 20.82 & 13.05 & 11.72 & 14.54 \\
\hline$M a x^{2} \mathrm{dE}^{*} a b$ & 30.04 & 57.99 & 41.09 & 38.09 & 56.03 & 35.22 & 17.58 & 40.32 \\
\hline \multicolumn{9}{|c|}{ Tones only } \\
\hline$M x^{1} \mathrm{dE}^{*} \mathrm{ab}$ & 11.47 & 13.84 & 9.82 & 10.91 & 13.72 & 13.17 & 11.26 & 14.10 \\
\hline $\operatorname{Max}^{2} \mathrm{dE}^{*} \mathrm{ab}$ & 20.97 & 19.71 & 15.86 & 18.67 & 18.45 & 20.12 & 16.10 & 20.10 \\
\hline
\end{tabular}


Table 2: The colorimetric accuracy of the profiled module Natural HB of the JVC DLA-RS15 digital projector, evaluated with Color Checker Classic chart, measured with PR-650; yellow colour marks the $d E^{*}$ ab values, lower than 5

\begin{tabular}{|c|c|c|c|c|}
\hline \multirow{3}{*}{ Color Checker Classic } & \multicolumn{4}{|c|}{$\Delta E^{*} a b ; P R-650$} \\
\hline & Natural HB & Natural HB & Natural HB & Natural HB \\
\hline & Without profile & ¡1ProBeamer & $\begin{array}{c}\text { basICCcolor display } \\
4.1 .22+\text { i1Pro }\end{array}$ & $\begin{array}{l}\text { basicColor display } \\
4.1 .22+\text { Spyder } 3)\end{array}$ \\
\hline Patch 1 ( Dark skin) & 4.03 & 8.32 & 7.03 & 7.43 \\
\hline Patch 2 ( Light skin) & 5.23 & 14.41 & 13.52 & 14.40 \\
\hline Patch 3 ( Blue Sky) & 9.90 & 11.45 & 10.12 & 10.60 \\
\hline Patch 4 ( Olive Green) & 9.44 & 10.00 & 8.49 & 9.00 \\
\hline Patch 5 ( Violet) & 8.99 & 12.54 & 11.08 & 11.68 \\
\hline Patch 6 ( Bluish Green) & 10.94 & 17.49 & 16.08 & 16.80 \\
\hline Patch 7 (Orange) & 20.19 & 13.82 & 13.62 & 13.90 \\
\hline Patch 8 (Purplish Blue) & 10.25 & 10.79 & 9.94 & 10.02 \\
\hline Patch 9 ( Moderate Red) & 7.51 & 11.43 & 10.23 & 11.03 \\
\hline Patch 10 ( Purple) & 13.38 & 7.13 & 5.79 & 6.42 \\
\hline Patch 11 ( Yelow Green) & 18.52 & 16.79 & 15.68 & 16.09 \\
\hline Patch 12 (Orange Yellow) & 31.80 & 16.97 & 17.20 & 17.55 \\
\hline Patch 13 (Blue) & 14.76 & 9.13 & 8.21 & 7.98 \\
\hline Patch 14 ( Green) & 13.97 & 13.36 & 11.85 & 12.47 \\
\hline Patch 15 ( Red) & 6.97 & 8.71 & 7.56 & 7.77 \\
\hline Patch 16 (Yelow) & 41.09 & 30.64 & 24.67 & 27.36 \\
\hline Patch 17 ( Magenta) & 12.15 & 12.23 & 11.29 & 11.82 \\
\hline Patch 18 ( Cyan) & 6.64 & 10.60 & 9.42 & 9.69 \\
\hline Patch 19 ( White) & 15.86 & 23.69 & 22.93 & 22.85 \\
\hline Patch 20 ( Neutral 8) & 10.98 & 18.98 & 18.30 & 18.08 \\
\hline Patch 21 ( Neutral 6,5) & 10.22 & 14.72 & 13.83 & 13.94 \\
\hline Patch 22 ( Neutral 5) & 8.88 & 11.05 & 9.85 & 10.28 \\
\hline Patch 23 ( Neutral 3,5) & 6.79 & 8.44 & 6.67 & 6.95 \\
\hline Patch 234 ( Black) & 6.21 & 5.76 & 4.70 & 5.03 \\
\hline \multicolumn{5}{|c|}{ All colours } \\
\hline$M x^{1} d E^{*} a b$ & 12.69 & 13.27 & 12.00 & 12.46 \\
\hline $\mathrm{Max}^{2} \mathrm{dE}^{*} \mathrm{ab}$ & 41.09 & 30.64 & 24.67 & 27.37 \\
\hline \multicolumn{5}{|c|}{ Tones only } \\
\hline$M x^{1} d E^{*} a b$ & 9.82 & 13.77 & 12.72 & 12.85 \\
\hline $\mathrm{Max}^{2} \mathrm{dE}^{*} \mathrm{ab}$ & 15.86 & 23.69 & 22.93 & 22.85 \\
\hline $\begin{array}{l}{ }^{1} M x \text { - average value, } d E^{*} a \\
{ }^{2} M a x \text { - maximum value, } d E\end{array}$ & 10. & & & \\
\hline
\end{tabular}


Table 3: The colorimetric accuracy of the profiled module Natural HB of the JVC DLA-RS15 projector, evaluated with Color Checker Classic chart, measured by i1Pro.

\begin{tabular}{|c|c|c|c|c|c|c|}
\hline \multirow[b]{3}{*}{ Color Checker Classic } & \multicolumn{6}{|c|}{$\Delta E^{*}$ ab; i1Pro } \\
\hline & Natural & Natural HB & Natural HB & Natural HB & Natural HB & Natural HB \\
\hline & $\begin{array}{l}\text { Without } \\
\text { profile }\end{array}$ & $\begin{array}{l}\text { Without } \\
\text { profile }\end{array}$ & ¡1ProBeamer & $\begin{array}{c}\text { Spyder3Elite } \\
4.0 .2\end{array}$ & $\begin{array}{c}\text { basICCcolor } \\
\text { display } \\
4.1 .22+ \\
\text { i1Pro } \\
\end{array}$ & $\begin{array}{c}\text { basicColor } \\
\text { display } \\
4.1 .22+ \\
\text { Spyder3) } \\
\end{array}$ \\
\hline Patch 1 (Dark skin) & 8.44 & 11.36 & 13.99 & 13.99 & 12.07 & 12.77 \\
\hline Patch 2 (Light skin) & 10.94 & 14.49 & 17.96 & 19.44 & 17.15 & 18.06 \\
\hline Patch 3 (Blue Sky) & 9.54 & 10.11 & 13.85 & 14.83 & 13.17 & 13.63 \\
\hline Patch 4 (Olive Green) & 10.32 & 11.69 & 13.34 & 12.23 & 11.66 & 12.53 \\
\hline Patch 5 (Violet) & 9.30 & 10.43 & 15.80 & 16.41 & 14.54 & 15.14 \\
\hline Patch 6 (Bluish Green) & 11.88 & 10.53 & 18.96 & 19.95 & 18.56 & 19.26 \\
\hline Patch 7 (Orange) & 18.65 & 19.60 & 17.15 & 17.11 & 17.05 & 17.39 \\
\hline Patch 8 (Purplish Blue) & 9.27 & 9.84 & 16.57 & 18.26 & 14.88 & 14.95 \\
\hline Patch 9 (Moderate Red) & 8.79 & 13.40 & 16.36 & 18.72 & 15.25 & 16.17 \\
\hline Patch 10 (Purple) & 11.20 & 13.86 & 12.03 & 11.53 & 10.42 & 11.91 \\
\hline Patch 11 (Yelow Green) & 16.88 & 17.10 & 19.39 & 21.02 & 19.08 & 19.38 \\
\hline Patch 12 (Orange Yellow) & 24.99 & 30.12 & 19.65 & 20.59 & 19.71 & 20.16 \\
\hline Patch 13 (Blue) & 10.42 & 11.35 & 15.52 & 17.19 & 14.05 & 13.43 \\
\hline Patch 14 (Green) & 11.93 & 12.30 & 15.28 & 17.76 & 14.53 & 15.39 \\
\hline Patch 15 (Red) & 10.88 & 9.03 & 14.13 & 16.53 & 12.25 & 13.20 \\
\hline Patch 16 (Yelow) & 35.22 & 40.23 & 24.87 & 29.97 & 26.37 & 29.04 \\
\hline Patch 17 (Magenta) & 10.34 & 15.33 & 16.72 & 19.21 & 16.31 & 16.79 \\
\hline Patch 18 (Cyan) & 5.30 & 13.56 & 12.96 & 13.73 & 11.84 & 12.30 \\
\hline Patch 19 (White) & 20.12 & 20.10 & 26.29 & 24.59 & 25.88 & 26.12 \\
\hline Patch 20 (Neutral 8) & 13.51 & 13.86 & 21.8 & 21.57 & 22.18 & 21.38 \\
\hline Patch 21 (Neutral 6,5) & 10.60 & 12.07 & 17.15 & 18.78 & 16.75 & 17.20 \\
\hline Patch 22 (Neutral 5) & 9.12 & 10.74 & 14.64 & 13.80 & 12.87 & 13.54 \\
\hline Patch 23 (Neutral 3,5) & 8.97 & 11.07 & 11.93 & 11.51 & 10.55 & 11.50 \\
\hline Patch 24 (Black) & 16.67 & 16.76 & 22.26 & 18.87 & 11.32 & 14.84 \\
\hline \multicolumn{7}{|c|}{ All colours } \\
\hline$M x^{1} d^{*} a b$ & 13.05 & 14.54 & 17.02 & 17.84 & 15.73 & 16.50 \\
\hline $\operatorname{Max}^{2} \mathrm{dE}^{*} a b$ & 35.22 & 40.32 & 26.29 & 29.97 & 26.37 & 29.04 \\
\hline \multicolumn{7}{|c|}{ Tones only } \\
\hline$M x^{1} d^{*} a b$ & 13.17 & 14.10 & 18.97 & 18.29 & 16.44 & 17.43 \\
\hline $\operatorname{Max}^{2} \mathrm{dE}^{*} a b$ & 20.12 & 20.10 & 26.29 & 24.59 & 25.88 & 26.12 \\
\hline
\end{tabular}




\section{CONCLUSION}

The control and evaluation of the projection, based on chromaticity, gradation, channel, grey balance, and colour gamut according to the ISO 12646:2010 standard is quite complicated and time-consuming. Choosing a suitable default module, whether for direct projection or for color management, is a major problem. Given that semi-professional and professional projectors are very expensive devices, the answer perhaps lies in colour management with hardware calibration and profiling - as is the case with better displays - and not in the set of (inappropriate) projection modules and/or settings with which we can experiment without achieving good results.

On the other hand, any colour management of digital projections will be effective and meaningful only if it will provide similar quality or colour differences as colour display on certified displays in accordance with ISO 12646: 2010 standard. A good channel balance and a very large colour range of saturated base colours do not yet provide a high-quality colour display in RGB colour spaces, in our case sRGB. The screen brightness (LV) of the white tile, which defines a certain colour space, must be obtained by all means. With the existing equipment, colorimetric analysis and profiling studio projectors in the selected projection module and colour space are the only reasonable choice. It can be summarized that the digital projection in displaying the standardized SRGB colour space is not comparable to high-quality CRT or LCD computer displays.

By profiling of the JVC DLA-RS15 digital projection, we cannot achieve the desired colour differences between the displayed and the reference values, as they are on average about twice or approximately three times the size of the allowed ones. Nevertheless, with the profiling by using each combination of measuring and software equipment, a significant reduction in maximum deviations is achieved. That is precisely the reason why we do our best when profiling a digital projection in studio conditions.

\section{REFERENCES}

[1] Ashdown M., Sato, Y.: "Steerable projector calibration", Computer Vision and Pattern Recognition Workshops, 2005. doi: 10.1109/CVPR.2005.533.

[2] Cooper, K.: "Northlight images", Northlight-images, URL: http://www.northlightimages.co.uk/reviews/profiling/i1-beamer.html (last request: 2018-05-09).

[3] Feierman, A.: "JVC DLA-RS15 projector review", Projectorreviews, URL: http://www.projectorreviews.com/jvc/dla-rs15/index.php (last request: 2018-09-10).

[4] Matt, S.: "Gut getroffen: Kalibrierung mit Eye-One Beamer", 86 - 88, 2003.

[5] Park, S.Y., Park, G. G.: "Active calibration of camera-projector systems based on planar homography", 20th International Conference on Pattern Recognition, ICPR 2010, (IAPR, Istanbul, Turkey, 2010). doi: 10.1109/ICPR.2010.87

[6] Turk, J.: "JVC dla-rs15 and rs25...a first look! jvc dla-rs15 projector", Avsforum, URL: http://www.avsforum.com/avs-vb/showthread.php?t=1174409 (last request: 2018-05-01).

[7] Wikipedia, Image projector, Wikipedia, URL: http://en.wikipedia.org/wiki/Image_projector (last request: 2018-09-09).

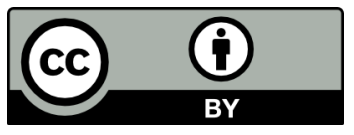

(C) 2018 Authors. Published by the University of Novi Sad, Faculty of Technical Sciences, Department of Graphic Engineering and Design. This article is an open access article distributed under the terms and conditions of the Creative Commons Attribution license 3.0 Serbia (http://creativecommons.org/licenses/by/3.0/rs/). 